\title{
Examining The Effectiveness Of Interventions Designed To Increase Mammography Adherence Among African American Women
}

Adam P. Knowlden, University of Cincinnati, USA Manoj Sharma, University of Cincinnati, USA

\begin{abstract}
The objective of this paper was to assess and synthesize the key findings, conclusions, and recommendations of mammography interventions targeting African American women conducted between 1999 and 2010. Collection of materials for this study included searches of academic databases using the following inclusion criteria: 1) publication in the English language, 2) between 1999 and 2010,3) conducted in the United States, 4) targeting African American women. Titles and abstracts of identified studies were evaluated independently by two researchers.

A total of 24 studies met the inclusion criteria. Interventions were categorized as either practicebased or community-based. Classifications were then sub-categorized based on the employment of targeted or tailored strategies.

Culturally-appropriate tailored and targeted messages are an effective approach to increase screening mammography adherence. Community-wide interventions that employ lay health advisors were found to assist in offsetting issues related to trust and access. Interventions delivered in faith-based settings were effective mediums for increasing adherence to screening guidelines. Stepped-care interventions were an efficient, cost-effective method for increasing adherence among non-compliant populations. The majority of the identified studies relied upon theoretical frameworks to guide the intervention. Community-based interventions should progress from atheoretical to theory-based intervention frameworks.
\end{abstract}

Keywords: Breast Cancer; Mammography; African American Women; Intervention

\section{INTRODUCTION}

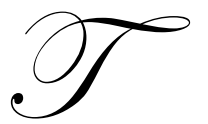

he effectiveness of mammograms to detect early stage breast carcinoma and decrease breast cancer mortality rates among women ages 40 to 70 has been well documented (National Cancer Institute, 2010). Despite the benefits of regular mammograms, mortality rates associated with breast cancer remain disproportionately high among African American women. Research suggests this disparity is partially due to late stage diagnosis and treatment (American Cancer Society, 2009). Public health interventions have attempted to close the gap in breast cancer stage of diagnosis by increasing mammogram adherence. The purpose of this study was to assess and synthesize the key findings, conclusions, and recommendations of mammography interventions targeting African American women conducted between 1999 and 2010.

Collection of materials for this study included searches of Academic Search Premier, CINAHL Plus with Full Text, ERIC, MEDLINE with Full Text, and Psychology and Behavioral Sciences Collection. In researching articles for this study, Boolean methodology was applied to the following search criteria within the specified databases: African American, mammography, breast cancer, intervention, determinants, and program. Titles and 
abstracts of identified studies were evaluated independently by two researchers using the following inclusion criteria: 1) publication in the English language, 2) publication between 1999 and 2010, 3) intervention conducted in the United States, 4) interventions targeting African American women. A total of 24 studies met the aforementioned inclusion criteria.

Within the context of mammography adherence, interventions can be classified as either community-based or practice-based (Paskett, 1999). Community-based mammography interventions include engaging the 1) community as a setting, as an 2) agent, as a 3) target, or as a 4) resource to carry out the intervention's objectives (McLeroy et al., 2003). Examples of interventions of this type include the employment of lay health advisors or church-based initiatives to promote mammography adherence (West et al., 2004). Practice, or clinic-based, interventions are based on the medical model and can be categorized as 1) mailed letters or postcard reminders to patients, 2) in-clinic physician reminders or staff education, 3) telephone counseling, and 4) stepped-care approaches (Paskett, 1999). For the purposes of this study, the interventions reviewed in this research report were categorized based on their classification as either practice-based or community-based programs. These general classifications were then sub-categorized based on whether the intervention employed targeted or tailored intervention strategies.

\section{RESULTS}

A variety of practice-based and community-based intervention designs have assessed the potential for interventions to increase mammography adherence. Tailored interventions are customized to beliefs, stages of change, and/or demographic characteristics. Tailored programs are delivered in person, in print, by telephone, or via technology such as kiosks or computers. Fundamentally, tailored interventions employ behavioral construct tailoring. Kreuter et al. (2005) described behavioral construct tailoring as the customization of health messages based upon participant responses. Alternatively, targeted interventions are generic in nature and attempt to address common factors among population subgroups. Videos, mass media campaigns, or print formats are the primary delivery formats of targeted interventions. In the identified interventions, effective targeted and tailored programs relied upon culturally-relevant focus groups to develop the program's materials and to ascertain the unique barriers the participants were likely to encounter.

\section{Practice-Based Targeted Interventions}

Audio-visual presentations offer the ability to convey information in a graphical context. Avis, Smith, Link, and Goldman (2004) compared the effectiveness of videotapes to a standardized pamphlet for the medium's potential to increase mammography screening adherence. The team developed a video tape documentary which contained interviews of women of diverse ethnic backgrounds, as well as a leading medical oncologist, and a demonstration of a woman receiving a mammogram. Baseline and follow up surveys administered at two and 12 months post-intervention revealed the videotape medium was slightly more effective $(80.4 \%)$ than the pamphlet (74.8\%) at increasing mammography screening. The researchers concluded that videotapes are a convenient, costefficient medium for increasing mammography rates.

Ostensibly, it may appear that audio-visual mediums offer a greater potential to generate affective responses than print mediums. Grindel, Brown, Caplan, and Blumenthal, (2004) examined the effect breast cancer screening message tone had on mammogram screening adherence. Video messages with three distinct milieus were evaluated; a positive/upbeat mode, a negative manner playing on fear of cancer, and a neutral/cognitive approach. Data were collected at baseline, post-test, and 12 months post-intervention. The varied tones of the videos did not make a significant difference in mammography screening rates, knowledge, or attitude of the participants. The researchers concluded that both the content and context of breast health communication messages may be more important than the tone in which the message is delivered.

\section{Practice-Based Tailored Interventions}

Telephone counseling allows researchers to employ direct health counseling tactics on a large scale. Allen and Bazargan-Hejazi (2005) assessed the effectiveness of culturally and ethnically tailored telephone interventions to increase mammography screening rates. Telephone scripts were developed based upon focus group findings. 
African American health counselors were trained to deliver the scripted telephone protocols. Counselors were specifically trained to identify and overcome mammography barriers unique to each participant. At six months postintervention, the intervention group reported $8 \%$ higher mammography screening rates than women in the control group.

Various mediums of tailored communication have been assessed for their efficacy to increase mammography adherence. Champion, Skinner, Menon, Seshadri, Anzalone, and Rawl (2002) conducted a study examining the impact of tailored mammography interventions in a factorial design. The investigators compared differences among four interventions groups: usual care, tailored telephone counseling for mammography, tailored mailed materials promoting mammography, and a combination of tailored mail and telephone counseling. When assessed at two months post-intervention, the combination tailored mail and telephone counseling intervention produced the greatest odds of increasing mammography adherence. Interestingly, the researchers noted that for women living without partners, all forms of tailored interventions tested in the study increased mammogram adherence.

Although cultural sensitivity is fundamental to breast cancer interventions targeting African American women, a lack of evidence for this underpinning prompted Kreuter et al. (2005) to investigate the impact of tailored diversity strategies on mammogram adherence. The investigators compared the effect of women's health magazines developed according to behavioral construct tailoring (BCT), culturally relevant tailoring (CRT) or both (BCT + CRT). Behavioral constructs employed included knowledge, beliefs, perceived barriers, stage of readiness, selfefficacy, and past behavior. Cultural constructs incorporated in the magazines included religiosity, collectivism, racial pride, and time orientation. Participants were assigned to BCT, CRT, BCT + CRT, or delayed/usual care. Participants receiving BCT + CRT magazines were more likely than those in the other groups to report getting a mammogram. This group also showed evidence of increased fruit and vegetable intake. The researchers concluded that incorporating culture into tailored cancer prevention and control interventions may increase their effectiveness in diverse populations.

Computer algorithms offer the potential to provide enriching interactive, tailored intervention experiences. However, those in greatest need of such delivery mediums often have limited access to advanced technology. In an attempt to bridge this digital divide, Kreuter et al. (2006) employed community kiosks to evaluate mammography knowledge and barriers in five community settings. Kiosks were used 4,527 times in 470 kiosk days at 40 different host sites. On average, users answered 4.3 out of 8 knowledge questions correctly. The team found that computer kiosks strategically positioned in community-settings can aid in disseminating information to low-income populations. In terms of usage, Laundromats averaged more uses per day than the other locations tested in this intervention. One explanation proposed by the researchers is the tendency for Laundromats to remain open seven days per week. Furthermore, Laundromat participants were also found to have the greatest need for breast cancer and mammography information. Interestingly, participants who used the kiosks located in churches answered more questions correctly and were more likely to complete the program; supporting the positive association between religiosity and health noted across numerous health fields.

\section{Practice-Based, Physician-Oriented, Tailored Interventions}

Increasing the efficacy of medical professionals to motivate women to participate in mammography is a front-line approach to reducing mortality rates associated with breast cancer. Coleman et al. (2003) tested the impact of a multi-method breast cancer screening intervention designed for rural healthcare providers. The researchers employed standardized patients to assess healthcare professionals' performance in conducting breast health interviews, performing clinical breast exam [CBE], and teaching breast self-exam [BSE]. Healthcare providers' knowledge and attitude of breast cancer screening significantly improved as a result of the intervention. Based on the study's findings, the researchers concluded that interventions designed to maximize the role of physicians in motivating women to have mammograms, participate in CBE, and practice BSE should be utilized.

A physician referral to undertake a mammogram has the potential to increase mammography compliance. However, the referral process may deter some women who would otherwise adhere to mammography if provided direct access. Simon, Gimotty, Moncrease, Dews, and Burack (2001) set out to determine if direct access to 
mammography services impacts adherence among low-income women. Participants were randomly assigned to one of three groups. The first group received a letter conveying that they were due for a screening mammogram. Participants in this group were instructed to visit their primary care physician who could provide a mammogram referral. The second letter removed the referral process and offered the participants direct access to mammography. Both groups that received letters were informed that the mammography would be provided at no out-of-pocket expense. The final group acted as the control group and received no letter. The researchers hypothesized that the letters allowing for patient-initiated mammography appointments would increase mammogram adherence. However, the researchers found no significant difference between the three groups. The researchers noted their intervention materials were not designed to address concerns and barriers specifically relevant to the target population.

Paskett et al. (1999) undertook a unique clinic-based mammography intervention incorporating components of community-based programs. The Forsyth County Cancer Screening project was designed to increase mammography and Pap smear screening rates among low-income, predominately African American, residents. The research team recruited a team of 174 physicians and residents to participate in the intervention. The project also included a consortium of community agencies to provide educational materials and referral services. Samples obtained from the intervention and comparison cities were collected at 3 -years post-intervention. Adherence rates in the intervention city increased from $31 \%$ to $56 \%$. However, in the comparison city, a non-significant increase from $33 \%$ to $40 \%$ was observed. The research team found that a combination of community outreach and clinic-based inreach strategies can increase mammography adherence. They also recommended that multiple behavior theories be incorporated during the development of the intervention framework.

\section{Combined Practice-Based Targeted/Tailored Interventions}

Determining whether targeted or tailored interventions produce greater results is a subject of on-going debate among researchers. Champion et al. (2006) contrasted the effectiveness of tailored and targeted mammography interventions. Three interventions including a tailored interactive computer-assisted instruction program as well as targeted video and pamphlet programs were contrasted. Each intervention was assessed for adherence and stage of readiness. At six months post-intervention the interactive computer-assisted instruction program produced the highest adherence rates $(40.0 \%)$ compared to targeted video (24.6\%) and pamphlet groups $(32.1 \%)$. In addition, participants in the tailored interactive group demonstrated more significant forward movement in their stage of mammography readiness (52\%) compared to the video (31.3\%) and pamphlet groups (46.4\%). The researchers speculated the forced engagement required of the interactive intervention caused participants to elicit greater concentration than the non-interactive approaches, leading to increased retention.

\section{Community-Based Targeted Interventions}

One of the appealing aspects of targeted interventions is their potential cost-effectiveness. Establishing a point of diminishing returns of targeted interventions has been an ongoing pursuit among researchers. Husaini et al. (2002) implemented a health education intervention in community settings utilizing a full program, a partial program, and a control group. The full program included a video presentation, a question and answer forum, and direct professional guidance. The partial program contained only a video segment while the control group received no education. Each group was assessed at three-months post-intervention. Interestingly, the researchers reported that the partial program was equally as successful as the full program in motivating participants to obtain a mammogram. The researchers concluded that partial programming initiatives could assist in promoting mammography while also controlling costs. The team also found residential communities, such as low-income housing projects, orient women into close-knit social networks. The researchers recommended that public health officials engage and incorporate these networks to maximize the impact of health education interventions.

Churches have remained a popular candidate for intervention settings. Holt and Klem (2005) compared the communication effectiveness of a secular and spiritually-oriented breast cancer educational booklet. The spirituallyoriented booklet contained biblical scriptures and religious/spiritual themes designed to encourage breast cancer screening. Six churches were randomly assigned to receive either secular or spiritually-oriented interventions. At one-month post-intervention respondents participated in a follow-up telephone interview. The spiritually oriented 
booklet provided a greater increase in mammography knowledge. When conducting church-based mammography programs, spiritually-oriented interventions may be more effective than secular interventions.

\section{Community-Based Tailored Interventions}

Lay health advisor (LHAs)-also referred to as community health advisors (CHAs), peer volunteers, peer educators, lay health educators, or lay community health workers-play an integral role in tailored community-based interventions. LHAs effectively serve as links between the professional health care system and the community (Earp et al., 2002). Typically, LHAs are recruited from the local community represented by the intervention. Subsequently, LHAs provide the characteristics inherent in a natural-helper model. This type of model differs from other types of volunteer models in three ways (Mayo, Sherrill, Crew, Watt, \& Mayo, 2004). Primarily, LHAs are perceived as community role models who have genuine empathy for community members. As opposed to volunteers brought in from outside of the community, LHAs are viewed as peers and thus are more adept to engage intervention participants and assist them in overcoming community-specific barriers. Secondly, LHAs are able to interact within the community's social networks spontaneously and informally. Such connectivity offers to the potential for the intervention to have an on-going, spill-over effect in the community (Earp et al., 2002). Finally, as LHAs are entrenched in the community's social networks, they possess the unique ability to influence beliefs, attitudes, and behaviors of numerous community social groups.

Researchers have noted that LHAs are in a unique position to access medically underserved populations. Mayo et al. (2004) assessed the impact of LHAs as part of the Avon Health Connector project; an undertaking designed to connect medically underserved women over 40 to culturally competent breast and cervical cancer education and screening. Data for the study was collected from a convenience sample of participants. Results indicated $69 \%$ of the individuals did not have health insurance, validating the program's effectiveness in reaching the medically underserved. Interestingly, $19 \%$ of participants had heard about the program through a friend of family member indicating the strength of community network and validating the effectiveness of LHAs in connecting women to breast health education and screening services.

As part of the North Carolina Breast Cancer Screening Program, Earp et al. (2002) evaluated the effectiveness of a lay health advisor network for promoting mammography screenings among low income, African American rural populations. The team assessed the reach, efficacy, and effectiveness of the initiative. After two years, the program's measures were associated with a 6 percentage point increase in community-wide mammography use. Furthermore, women in the low-income sub-group displayed an 11 percentage point increase. The substantial increase was attributed to the success of the LHA network, demonstrating the value of LHAs as mediums for promoting mammography screenings among low income, rural populations.

Paskett et al., (2006) conducted a randomized trial to test the impact of a LHA-oriented intervention among minority, rural women. In their study, Paskett and colleagues provided an overview of the LHA training protocol developed for the intervention. The effectiveness of the LHA training and study design was evident by the project's findings. At 12 and 14 months post-intervention, $42.5 \%$ of the LHA group participants received a mammography compared to only $27.3 \%$ in the control group. In addition, the LHA group displayed higher belief scores and reduced barriers.

Training LHAs to successfully engage and convince community members to engage in mammography is critical to the success of the program. Fowler, Rodney, Roberts, and Broadus (2006) delineated the specifics of the training program they developed as part of a collaborative breast health intervention. The research team developed a 15-hour, modular training program consisting of team building an overview of the intervention deliverables. Although only 68 of the 90 participants received a mammography, the researchers noted a significant improvement of knowledge scores of the participants. Further, they noted LHAs are instrumental in reducing barriers specific to African American women.

Kidder's (2008) analysis of the Protect Our Women intervention revealed that community leaders are in a unique position to offset barriers frequently associated with mammograms. The intervention was designed to address inadequate outreach, inappropriate educational materials, and distrust of the health care system. As part of the 
intervention, a convenience sample of women was identified by a panel of community leaders. At the conclusion of the program, $79 \%$ of the participants who completed the breast awareness workshop obtained mammograms, with 9\% having mammograms scheduled at 1-month post-intervention. Kidder concluded that including prominent community leaders in the intervention recruitment process can assist in offsetting common barriers to mammography including mistrust of the health care system, health beliefs, and access.

Increasing mammography use among African American women ages 65 and older has received minimal attention. To address this issue, Zhu et al. (2002) conducted an intervention targeting older African American women with single marital status. Ten public housing complexes were randomly assigned to either an intervention or control group. The educational intervention was delivered by lay health educators (LHE). The LHEs were African American women of the same age range of the participants. The researchers assessed the effectiveness of the measured variables at baseline and at 1 and 2 years post-intervention. The team reported that the differences between control and experimental groups did not reach statistical significance. The researchers proposed that the average age of the participants (mean age $=76$ ) may explain the lack of mammography intervention effects. The team suggested elderly participants may be less interested in preventative measures and have poorer memories. They also proposed that booster sessions may be necessary for older, single African American women. For costeffectiveness, the team advocated shorter, more frequent booster sessions.

Addressing attitudes towards mammography is a consistent theme across community-based tailored interventions. A retrospective analysis of the Carolina Breast Cancer Screening Program conducted by Rauscher, Earp, and O'Malley (2004) found the greatest impact on attitude improvement was among participants without a recent mammogram at baseline and with the least positive baseline attitude scores. The team's findings suggest mammography interventions should focus on improving attitudes toward mammography rather than attitudes about breast cancer risk. Furthermore, programs should target women with the least acceptance and adherence rates as well as the and lowest awareness of mammography.

\section{Combined Targeted And Tailored Community-Based Interventions}

Garza et al. (2005) combined elements of tailored and targeted interventions to promote a no-cost mammography-screening program for low-income African American women. The intervention was implemented across four stages. Baseline assessment was implemented by community health workers (CHWs). During the home visit, the CHWs provided participants with two brochures. The first brochure was standard mammography educational literature. The second brochure described a breast cancer survivor story of a local community leader. The second stage consisted of the CHAs home visits in which the instrument was provided again to detect knowledge changes. During the visit the CHAs invited each participant to an educational session at a local church. The church educational session was conducted by the pastor, a medical provider, and a team of health educators. The fourth stage consisted of a mammogram appointment. The intervention achieved a 50\% screening rate, which the researcher attributed to a successful culturally targeted intervention with a spiritual component.

Powell et al. (2005) evaluated the effectiveness of a church-based breast cancer screening education program for women 40 years and older in rural Alabama. The project incorporated a community-based participatory research approach to develop the intervention. The selected churches were randomly assigned to three groups; full program, partial program and control. Both the full and partial programs consisted of an educational session held at the church. The session contained a video aimed at reducing fears and concerns of the mammography procedure as well as a question and answer follow-up session. In addition the full program participants received a home visit by a home health educator (HHE). The HHEs were responsible for reviewing the video material, provide selfexamination training, and assist in scheduling a mammogram. In addition, they were granted authority to provide transportation and babysitting services to eligible participants. The full program positively affected mammography attainment resulting in a 38\% increase in adherence from baseline. Church-based outreach programs offer several advantages when targeting hard to access populations. Primarily they build upon pre-existing social ties, fostering trust and reassurance of the delivered messages. Inherently, church-based interventions provide an appropriate cultural context assisting in overcoming barriers unique to the local community. Despite the advantages, the research team noted that rural, church-oriented outreach programs are labor intensive and difficult to implement due to communication and scheduling challenges. 


\section{Stepped-Care Interventions}

Stepped-care, or progressive, interventions integrate aspects of both targeted and tailored interventions. The most promising feature of this type is the cost efficient allocation of resources. With this approach, targeted measures, such as postcards, could be incorporated as a first-level intervention. Non-responders could then be contacted using tailored telephone counseling or lay health advisors as a second-level intervention; effectively reserving more costly approaches for non-compliant audiences. An additional facet of this intervention type is the potential for unique clinic-community partnerships.

West et al. (2004) evaluated a stepped-care community clinic-based mammography intervention targeted at low-income African American women. Stage one participants received either a personalized reminder letter or usual care. Participants that did not receive a mammogram by the 6-month follow up period were randomized into tailored telephone call or tailored letter intervention groups. No group differences were reported by the second, 6 month follow-up. Due to this finding, the researchers questioned whether the added personnel cost required of the telephone counseling justified the cost. However, the researchers did note that among women who had never received a mammogram, the tailored counseling did produce higher screening rates (16\%) compared to the tailored letter $(7 \%)$. The researchers concluded that stepped-care models are effective for concentrating resources and that tailored mammography promotion counseling may be effective for women who have never engaged in screening.

\section{Theory-Based Interventions}

A significant portion of the interventions $(n=16)$ relied upon theoretical frameworks to guide either the planning, implementation, and/or evaluation stages of the reviewed studies. Frequently, research teams employed an integrated approach, combining numerous models and theories to tap into specific behavioral and environmental constructs. A number of the reviewed studies $(n=8)$, particularly the community-based interventions $(n=5)$, either did not report or did not apply a theoretical framework. In order to reify theoretical frameworks it is critical that community-based researchers begin assessing the efficacy and effectiveness of health-related theoretical constructs.

The Health Belief Model [HBM] was the most frequently utilized model (n=9) employed in the reviewed interventions. The HBM addresses the value and expectations an individual places on a particular outcome. One of the inherent limitations of the HBM is its inability to address cultural and socio-economic factors (Sharma \& Romas, 2008), which may explain why it was often amalgamated with other behavior-related models.

The Transtheoretical Model (TTM), or stages of change model, was the second most $(n=6)$ popular model incorporated in the reviewed interventions. The TTM explains the process of behavior change. Interventions employing this model were designed to move participants forward in stages of mammography readiness and adherence. The Theory of Reasoned Action [TRA], Theory of Planned Behavior [TpB], Social Cognitive Theory [SCT], and Social Learning Theory [SLT] were also prevalent behavioral models employed in the reviewed interventions. The TpB is an extension of the TRA, including an additional construct. The goal of these two theories is to predict behavioral intention; the immediate antecedent of a given behavior. One of the salient limitations of this theory is that it assumes behaviors are guided by rational thought processes; however, fatalism, a common barrier of mammogram interventions, is an irrational fear that may confound the predictability of this theory. Care should be taken when employing this theory to ensure the intervention addresses fatalistic thoughts associated with mammograms.

The Social Learning Theory, later renamed the Social Cognitive Theory, assumes that human behavior is influenced by personal, environmental, and behavioral factors. One of the strongest components of this theory is the self-efficacy construct. Self-efficacy describes an individual's confidence to perform a given behavior. A general method for improving self-efficacy in the identified studies was the use of LHA's who were trained to serve as credible role models to participants. This theory is primarily useful for individuals prepared to initiate change and is not specifically designed to modify behaviors. In the referenced studies, this theory was often used in conjunction with other theories and models of behavior change. 


\section{CONCLUSION}

Interventions designed to increase breast cancer awareness and mammography screening adherence among African American women have resulted in varying levels of success. LHAs as well as physicians and nurses play an instrumental role in motivating African American women to participate in mammography screening. Lack of health insurance, limited knowledge, issues related to trust, fatalism, and access to services were the primary barriers identified for this population to participate in breast cancer screenings.

Culturally-appropriate tailored and targeted messages were an effective approach to increase mammography screenings. Community-wide interventions that employ lay health advisors were found to assist in offsetting issues related to trust and access. Culturally sensitive tailored messages and interventions delivered in faith-based settings or with a spiritual connotation were effective mediums for increasing adherence to screening guidelines. Stepped-care interventions were identified as an efficient, cost-effective method for increasing adherence among non-compliant populations. Tailored interventions were found to be more impactful than targeted interventions. The majority of the identified studies relied upon theoretical frameworks to guide the intervention and to evaluate the intervention objectives. Community-based interventions should progress from atheoretical to theorybased intervention frameworks.

\section{LIMITATIONS}

There are several limitations of this review. Primarily, this is a qualitative, narrative review and not a quantitative meta-analysis. Consequently, quantitative measures cannot be ascertained from this review. This review did not restrict studies based on research design, which may be considered a limitation by some researches. Finally, this review was limited to studies published in the English language and conducted in the United States, between 1999 and 2010, specifically targeting African American women. Additional studies may have been published since the time of this review which may support or contradict findings reported in this analysis.

\section{AUTHOR INFORMATION}

Adam P. Knowlden is a second-year Ph.D. student at the University of Cincinnati majoring in Health Promotion and Education. E-mail: knowldam@email.uc.edu

Manoj Sharma is a Professor of Health Promotion and Health Education and Public Health at the University of Cincinnati. He is a physician by initial training and has also completed his doctorate in Preventive Medicine/Public Health from The Ohio State University. His research interests are in designing theory-based health promotion programs. E-mail: manoj.sharma@uc.edu

\section{REFERENCES}

1. Allen, B. J., \& Bazargan-Hejazi, S. (2005). Evaluating a tailored intervention to increase screening mammography in an urban area. Journal of the National Medical Association, 97(10), 1350-1360. Retrieved from http://www.ncbi.nlm.nih.gov/pubmed/16353657

2. American Cancer Society. Breast cancer facts \& figures 2009-2010. Atlanta: American Cancer Society, Inc. Retrieved from http://www.cancer.org/acs/groups/content/@nho/documents/document/f861009final90809pdf.pdf

3. Avis, N. E., Smith, K. W., Link, C. L., \& Goldman, M. B. (2004). Increasing mammography screening among women over age 50 with a videotape intervention. Preventive Medicine, 39(3), 498-506. doi: 10.1016/j.ypmed.2004.05.024

4. Bazargan, M., Bazargan, S. H., Calderon, J. L., Husaini, B. A., \& Baker, R. S. (2003). Mammography screening and breast self-examination among minority women in public housing projects: The impact of physician recommendation. Cellular and Molecular Biology, 49(8), 1213-1218. doi: 10.1186/1472-6874-820 
5. Champion, V. L., Skinner, C. S., Menon, U., Seshadri, R., Anzalone, D. C., \& Rawl, S. M. (2002). Comparisons of tailored mammography interventions at two months postintervention. Annals of Behavioral Medicine, 24(3), 211-218. doi: 10.1207/S15324796ABM2403_06

6. Champion, V. L., Springston, J. K., Zollinger, T. W., Saywell, R. M., Jr., Monahan, P. O., Zhao, Q. Russell, K. M. (2006). Comparison of three interventions to increase mammography screening in low income African American women. Cancer Detection and Prevention, 30(6), 535-544. doi: 10.1016/j.cdp.2006.10.003

7. Coleman, E. A., Lord, J., Heard, J., Coon, S., Cantrell, M., Mohrmann, C., O’Sullivan, P. (2003). The Delta project: increasing breast cancer screening among rural minority and older women by targeting rural healthcare providers. Oncology Nursing Forum, 30(4), 669-677. doi: 10.1188/03.ONF.669-677

8. $\quad$ Earp, J. A., Eng, E., O'Malley, M. S., Altpeter, M., Rauscher, G., Mayne, L., Qaqish, B. (2002). Increasing use of mammography among older, rural African American women: results from a community trial. American Journal of Public Health, 92(4), 646-654. doi: 10.2105/AJPH.92.4.646

9. Fowler, B. A., Rodney, M., Roberts, S., \& Broadus, L. (2005). Collaborative breast health intervention for African American women of lower socioeconomic status. Oncology Nursing Forum, 32(6), 1207-1216.

10. Garza, M. A., Luan, J., Blinka, M., Farabee-Lewis, R. I., Neuhaus, C. E., Zabora, J. R., \& Ford, J. G. (2005). A culturally targeted intervention to promote breast cancer screening among low-income women in East Baltimore, Maryland. Cancer Control. Journal of the Moffitt Cancer Center, 12 Suppl 2, 34-41. Retrieved from http://www.moffitt.org/moffittapps/ccj/index.html

11. Grindel, C. G., Brown, L., Caplan, L., \& Blumenthal, D. (2004). The effect of breast cancer screening messages on knowledge, attitudes, perceived risk, and mammography screening of African American women in the rural South. Oncology Nursing Forum, 31(4), 801-808. doi: 10.1188/04.ONF.801-808

12. Holt, C. L., \& Klem, P. R. (2005). As you go, spread the word: spiritually based breast cancer education for African American women. Gynecologic Oncology, 99(3 Suppl 1), S141-142. doi: 10.1016/j.ygyno.2005.07.066

13. Hurd, T. C., Muti, P., Erwin, D. O., \& Womack, S. (2003). An evaluation of the integration of nontraditional learning tools into a community based breast and cervical cancer education program: The Witness Project of Buffalo. BMC Cancer, 3, 18. doi: 10.1186/1471-2407-3-18

14. Husaini, B. A., Sherkat, D. E., Levine, R., Bragg, R., Van, C. A., Emerson, J. S., \& Mentes, C. M. (2002). The effect of a church-based breast cancer screening education program on mammography rates among African-American women. Journal of the National Medical Association, 94(2), 100-106.

15. Kidder, B. (2008). P.O.W. (protect our women): Results of a breast cancer prevention project targeted to older African-American women. Social Work in Health Care, 47(1), 60-72. doi: 10.1080/00981380801970830

16. Kreuter, M. W., Sugg-Skinner, C., Holt, C. L., Clark, E. M., Haire-Joshu, D., Fu, Qiang, Booker, A. C., Steger-May, K., \& Bucholtz, D. (2005). Cultural tailoring for mammography and fruit and vegetable intake among low-income African-American women in urban public health centers. Preventive Medicine, 41(1), 53-62. doi:10.1016/j.ypmed.2004.10.013

17. Kreuter, M. W., Black, W. J., Friend, L., Booker, A. C., Klump, P., Bobra, S., \& Holt, C. L. (2006). Use of computer kiosks for breast cancer education in five community settings. Health Education \& Behavior, 33(5), 625-642. doi: 10.1177/1090198106290795

18. Mayo, R. M., Sherrill, W. W., Crew, L., Watt, P., \& Mayo, W. W. (2004). Connecting rural African American and Hispanic women to cancer education and screening: The Avon Health Connector project. Journal of Cancer Education. The Official Journal of the American Association for Cancer Education, 19(2), 123-126. doi: 10.1207/s15430154jce1902_14

19. McLeroy, K. R., Norton, B. L., Kegler, M. C., Burdine, J. N., \& Sumaya, C. V. (2003). Community-based interventions. American Journal of Public Health, 93(4), 529-533. Retrieved from http://ajph.aphapublications.org/cgi/content/full/93/4/529

20. National Cancer Institute: Breast Cancer Screening. Health professional. (September, 2010). Retrieved from http://www.cancer.gov/cancertopics/pdq/screening/breast/HealthProfessional 
21. Paskett, E. D., Tatum, C. M., D'Agostino, R., Rushing, J., Velez, R., Michielutte, R., \& Dignan, M. (1999). Community-based interventions to improve breast and cervical cancer screening: Results of the Forsyth County Cancer Screening (FoCaS) Project. Cancer Epidemiology, Biomarkers \& Prevention : A Publication of the American Association for Cancer Research, cosponsored by the American Society of Preventive Oncology, 8(5), 453-459. Retrieved from http://cebp.aacrjournals.org

22. Paskett, E. D., Tatum, C., Rushing, J., Michielutte, R., Bell, R., Foley, K. L., Reeves, K. (2006).

Randomized trial of an intervention to improve mammography utilization among a triracial rural population of women. Journal of the National Cancer Institute, 98(17), 1226-1237. doi: 10.1093/jnci/djj333

23. Powell, M. E., Carter, V., Bonsi, E., Johnson, G., Williams, L., Taylor-Smith, L., Husaini, B. A. (2005). Increasing mammography screening among African American women in rural areas. Journal of the Health Care Poor \& Underserved, 16(4 Suppl A), 11-21. doi: 10.1353/hpu.2005.0129

24. Rauscher, G. H., Earp, J. A. L., \& O'Malley, M. (2004). Relation between intervention exposures, changes in attitudes, and mammography use in the North Carolina Breast Cancer Screening Program. Cancer Epidemiology, Biomarkers \& Prevention: A Publication of the American Association for Cancer Research, cosponsored by the American Society of Preventive Oncology, 13(5), 741-747.

25. Sharma, M., \& Romas, J. A. (2008). Theoretical foundations of health education and health promotion. Sudbury, Massachusetts: Jones and Bartlett Publishers.

26. Simon, M. S., Gimotty, P. A., Moncrease, A., Dews, P., \& Burack, R. C. (2001). The effect of patient reminders on the use of screening mammography in an urban health department primary care setting. Breast Cancer Research and Treatment, 65(1), 63-70. doi: 10.1023/A:1006410711370

27. West, D. S., Greene, P., Pulley, L., Kratt, P., Gore, S., Weiss, H., \& Siegfried, N. (2004). Stepped-Care, Community Clinic Interventions to Promote Mammography Use among Low-Income Rural African American Women. Health Education \& Behavior: The Official Publication of the Society for Public Health Education, 31(4_suppl), 29S-44. doi: 10.1177/1090198104266033

28. Zhu, K., Hunter, S., Bernard, L. J., Payne-Wilks, K., Roland, C. L., Elam, L. C. Levine, R. S. (2002). An intervention study on screening for breast cancer among single African-American women aged 65 and older. Preventive Medicine, 34(5), 536-545. doi: 10.1006/pmed.2002.1016 